\title{
TRL-based Measurement of Embedded Circuits in Microwave Printed Circuit Boards Including Frequency Conversion
}

\author{
Petr OUREDNIK, Premysl HUDEC \\ Dept. of Electromagnetic Field, Czech Technical University, Technicka 2, 16627 Prague, Czech Republic \\ \{ouredpet, hudecp\}@fel.cvut.cz
}

Submitted June 16, 2018 / Accepted September 30, 2018

\begin{abstract}
The paper deals with the measurement of individual components or circuits embedded in more complex radio frequency $(R F)$ or microwave printed circuit boards (PCBS). Since no standard RF measurement enables the direct parallel connection of an analyzer to the boards being tested, individual components are often measured by destructively cutting manufactured boards and by attaching the RF connectors to the concerned parts. This article shows that this problem, thanks to suitable calibration standards that have been designed and manufactured, can be solved by vector measurements and a TRL calibration process. The measurements also work when the boards to be measured include frequency conversion. The applicability of the developed method has been verified by practical measurements and its accuracy influenced by variations of the parameters of the surrounding circuits has been investigated by an uncertainty analysis.
\end{abstract}

\section{Keywords}

Microwave measurement, calibration-correction techniques, TRL calibration, vector network analyzer, uncertainty analysis

\section{Introduction}

Traditionally, radio frequency (RF) and microwave systems (communication, radar) consist of a definite set of standard circuits (amplifiers, mixers, filters, dividers) interconnected at a single printed circuit board (PCB). For example, Fig. 1 depicts the transmitter (TX) part of a pseudo-noise continuous wave $(\mathrm{CW})$ radar sensor operated at $11 \mathrm{GHz}$. The sensor consists of monolithic microwave integrated circuits (MMICs) voltage control oscillator (VCO), amplifiers, mixers, frequency multipliers, microstrip filters and power dividers, and sections of interconnecting microstrip or grounded coplanar waveguide (GCPW) lines. During the manufacturing process, the parameters of all these components can be altered. The MMICs by the quality of the soldering, the microstrip filters and dividers by the etching process. The effects of the etching on impedance and crosstalk between tracks are evaluated in [1]. Therefore, it is advantageous, and sometimes necessary, to measure the resulting parameters of the aforementioned circuits, although this is not easily done in the RF and microwave frequency bands. An example of an EMI filter development based on information obtained by this measurement is shown in [2].

The need to measure individual components or circuits embedded in more complex system PCBs represents one of the most critical problems of RF and microwave design, manufacturing and measuring. Recently efforts have been made to perform such an in-circuit measurement. Majority of these methods were based on contactless approach, where fields are coupled via probes to the measurement instrument. The probes can be in the form of current probes as showed in [3] or composed as a direction coupler showed in [4] and [5]. To be able to measure on high frequencies by these methods a calibration needs to be performed beforehand. Measurement precision for all these methods depends on the repeatability of the probe positioning.

An example of this problem concerns the measurement of the band-pass filter (BPF) embedded in the radar TX described above, or in the common test up-converter module, as shown in Fig. 2. This test module consists of an RF mixer, a local oscillator, the BPF, and an output power amplifier (PA), all of which are assembled on a single

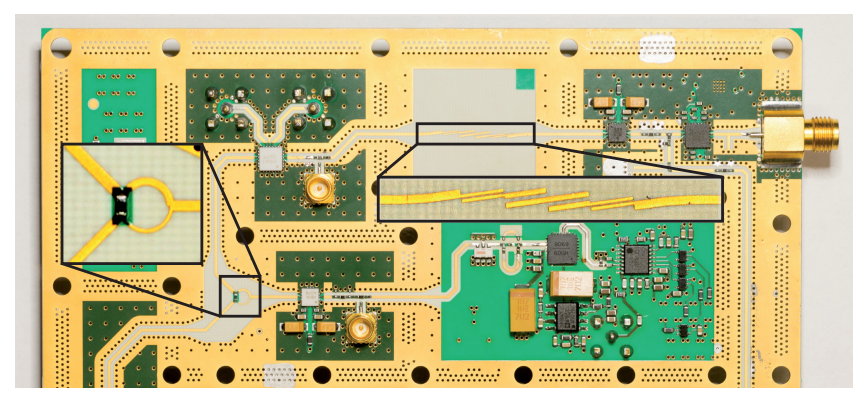

Fig. 1. $11 \mathrm{GHz}$ transmitter on microwave $\mathrm{PCB}$. 
PCB equipped only with input and output RF connectors. If filter parameters are to be measured, it is necessary to cut the filter from the board, thus rendering the board useless. At RF it is impossible to apply anything, such as the ideal voltagemeter (VM) widely used in low-frequency (LF) bands.

Evaluating the parameters of similar BPF at LF can be performed by a simple parallel connection of $2 \mathrm{VMs}$, as can be seen in Fig. 2. VM1 measures $V_{1}$ input voltage, while VM2 measures $V_{2}$ output voltage. Since both VMs show a magnitude of $Z_{\text {vin }}$ input impedances close to $\infty$ at LF, the measured structure is not loaded and influenced, and the concerned voltages are measured precisely. But at RF, a significantly different situation must be expected due to the always present $C_{\mathrm{p}}$ parasitic VMs capacitances which result in a dramatic $\left|Z_{\mathrm{vin}}\right|$ decrease. For example, even a very low $C_{\mathrm{p}}=1 \mathrm{pF}$ value causes $\left|Z_{\mathrm{vin}}\right|$ to drop to a mere $80 \Omega$ at $f=2 \mathrm{GHz}$. Such a low input impedance gives rise to voltage dividers which prevent both $V_{1}$ and $V_{2}$ from being measured precisely. Moreover, such complex loading can significantly disturb BPF's transmission parameters designed for operations with $Z_{0}=50 \Omega$ at both ports. That is why individual circuits and components embedded in the assembled RF and microwave PCBs cannot be measured directly. However, definite components, such as antennas or narrow-band filters, are sensitive structures and their performance can be significantly influenced by the manufacturing process which is why their final parameters should always be checked.

Reference [6] describes a new method based on a vector network analyzer (VNA) measurement and vector calibration/correction which enables the solution of the above mentioned problem. The method was developed for return loss (RL) measurement of passive radiating elements (patch antennas) embedded in more complex active antenna modules. The paper shows that by using a suitable set of standards and a thru-reflect-line (TRL) calibration/correction procedure, it is possible to measure directly inaccessible patch elements embedded in the concerned PCBs.

Positive results presented in [6] inspired an attempt to measure the BPF embedded in an up-converting module similar to that described in Fig. 2. The module includes a planar coupled-microstrip BPF that is sensitive to the manufacturing process, thereby requiring its final parameters (both transmission and reflections) to be checked. The task is significantly

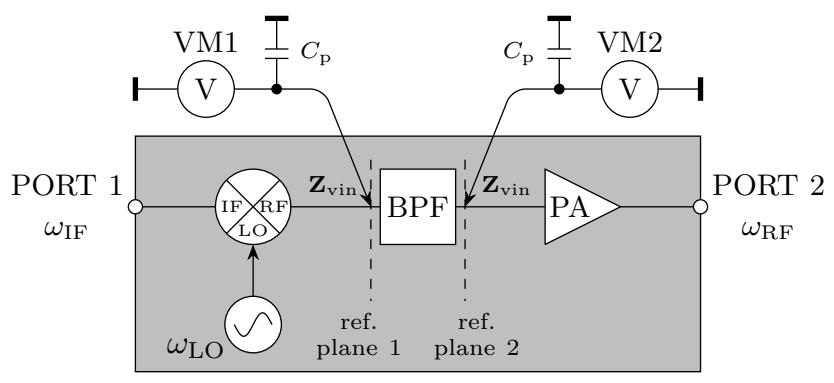

Fig. 2. Typical up-converting RF module. complicated by the fact that the structure includes frequency conversion. Using the local oscillator (LO), the signal with frequency $\omega_{\mathrm{IF}}$ at input PORT 1 is converted to an output signal with frequency $\omega_{\mathrm{RF}}$ at output PORT 2. It is, therefore, necessary to measure S-parameters of the embedded BPF with different frequencies at PORT 1 and PORT 2. It goes without saying that the PCB in question cannot be damaged in any way.

\section{TRL Calibration Technique}

The TRL techniques were developed to enable precise measurements when using vector network analyzers, see Fig. 3. Vector measurements of any device under test (DUT) are greatly influenced by measurement paths between the VNA ports and the DUT. The path between port P1 and the DUT can be described by an ERR-A error two-port, and the path between port P2 and the DUT by an ERR-B error two-port. If the S-parameters of both error two-ports are known, their influence can be eliminated by the correction process and the "clean" DUT S-parameters obtained.

To evaluate the S-parameters of both error two-ports during the calibration process, the TRL method employs the following standards: THRU (short interconnecting transmission line), REFLECT (shortened sections of transmission lines at each end), and LINE (transmission line with extended length). The necessary mathematical formulas can be found in [7], [10] and [11].

Compared to other calibration-correction methods, the main advantage of the TRL technique is its relative simplicity and extremely good definability of the standards employed. The method requires neither a problematic MATCH (precise $50 \Omega$ load) nor a similarly problematic OPEN (infinite impedance). First and foremost, it relies on transmission

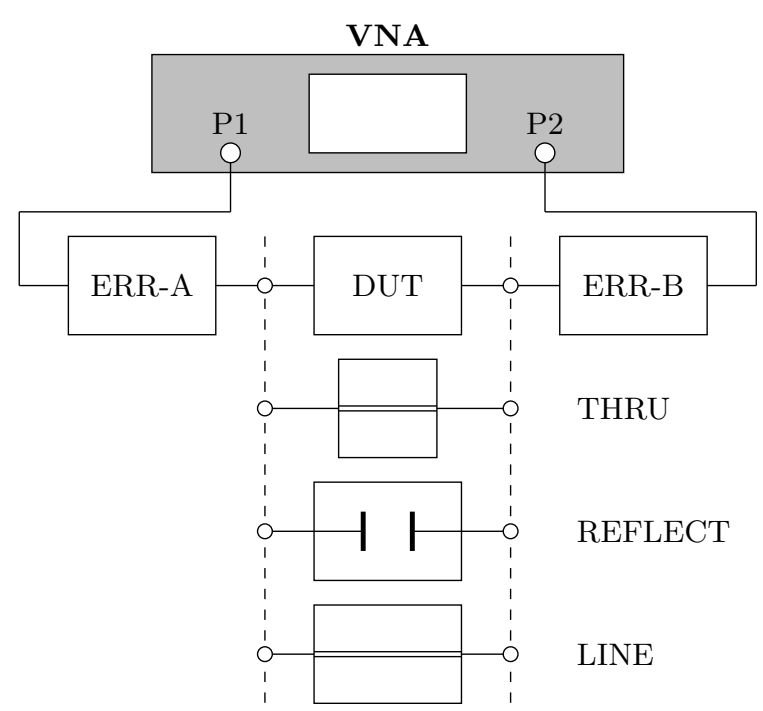

Fig. 3. VNA measurement using TRL calibration-correction techniques. 
lines with exact characteristic impedances and properly chosen lengths. One clear disadvantage is the complexity of the corresponding mathematical formulas which can, in some cases, complicate the application. Probably the most detailed description of the related mathematical formulas is found in [11]. The formulas are rather complex and extensive; in the reference in question they occupy nearly two standard text pages. If common computer processing is used, their complexity does not represent any significant problem. Difficulties can be met when an analytical treatment is required. In the given case, this concerns the evaluation of uncertainties using standard methods based on partial derivatives of the analytical formulas with respect to all variables which can be influenced by uncertainties. The TRL formulas are so complex that the evaluation of partial derivatives is not feasible and other techniques must be used.

The central idea of this method developed for the measurement of individual circuits embedded in complex microwave system PCBs is to include circuits surrounding the DUT into error two-ports, and to measure the DUT through outer board connectors. As this article demonstrates, the method can overcome even significant obstacles such as amplifiers (if they do not show extreme isolation) or frequency conversion performed within the concerned PCB. If embedded attenuations are too high, the T-method developed in [6] can be employed. In spite of extremely good results obtained using the TRL process, if beneficial, any similar VNA calibration-correction method can be used.

\section{Developed Measurement Method}

To evaluate the possibilities of the above described method for the measurement of 2-ports embedded in more complex up-converting (or down-converting) modules, a test board consisting of a $2.1 \mathrm{GHz}$ microstrip BPF and a mixer was designed and manufactured, see Fig. 4 and Fig. 5. Figure 4 also includes a description of a measurement setup developed, after several trials, for the measurement of S-parameters of the concerned RF structures containing frequency conversion.

The setup is based on a 2-port VNA and contains external mixers, a power divider and an LO. This setup for a measurement of frequency converting devices is deeply explained in [7], [8] and [9]. For $S_{21}$ and $S_{11}$ measurements, the VNA's internal LO1 was switched to PORT 1 and swept in a 0.55 to $1.55 \mathrm{GHz}$ frequency band. The external (or second internal, if available) LO2 was operated at a fixed frequency of $1.05 \mathrm{GHz}$. Using the external mixers, MX1 and MX2, the incident and reflected signals at PORT 1 were converted to a 1.6 to $2.6 \mathrm{GHz}$ frequency range and measured by the VNA's $a_{1}$ and $b_{1}$ receivers. The MXD mixer included in the test board also generates a signal in the 1.6 to $2.6 \mathrm{GHz}$ frequency range required for BPF's testing. The necessity to include AF auxiliary filters (high-pass, connected between MXD's RF port and the BPF) into all boards represent the only inconvenience of the developed measurement method. The main task of these filters is to reflect the 0 to $0.5 \mathrm{GHz}$ mirror signals back to the MXD mixer where they are again multiplied with the $1.05 \mathrm{GHz}$ LO2 signal. The resulting upconverted signals interfere with the PORT1 0.55 to $1.55 \mathrm{GHz}$ signals. In case all boards reflect the mirror signals with the same amplitude, and in the same plane, their influence can be eliminated in the calibration-correction process. That is why the identical AF filters must be included in all both test and calibration boards. They can be formed by the simple and small structure shown in Fig. 5.

For $S_{12}$ and $S_{22}$ measurements, the LO1 was switched to the PORT 2 branch, while its range was set to 1.6 to $2.6 \mathrm{GHz}$. To prevent mirror reception at the $\mathrm{a}_{1}$ and $\mathrm{b}_{1}$ receivers, an external $1.55 \mathrm{GHz}$ low-pass filter was connected between the BPF to be measured and the VNA. As already stated, the actual measurement of the embedded BPF is based on the TRL (THRU-REFLECT-LINE) calibration/correction method described in greater detail, e.g., in [7], [10] and [11]. That is why it was also necessary to design and realize the corresponding calibration boards (standards), see Fig. 6.

On the THRU board, the BPF is replaced by a direct thru connection. On the REFLECT board, there are 2 shorts (vias) situated in the BPF's reference planes. The LINE board includes a $50 \Omega$ microstrip line replacing the concerned BPF. Since mixers and the auxiliary high-pass filters are included in all boards, their influence can be removed by using the calibration-correction process and "clean" BPF S-parameters can be obtained. High variances of the parameters of circuits surrounding the DUT represent the main potential problem of this method.

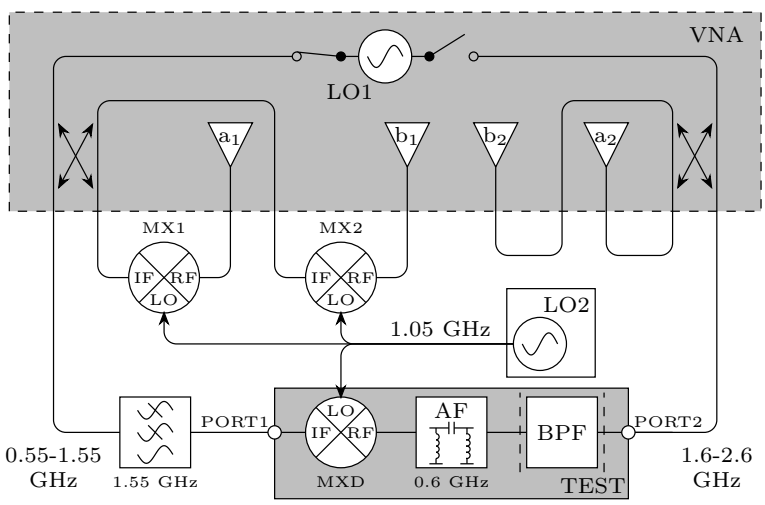

Fig. 4. Developed measurement setup.

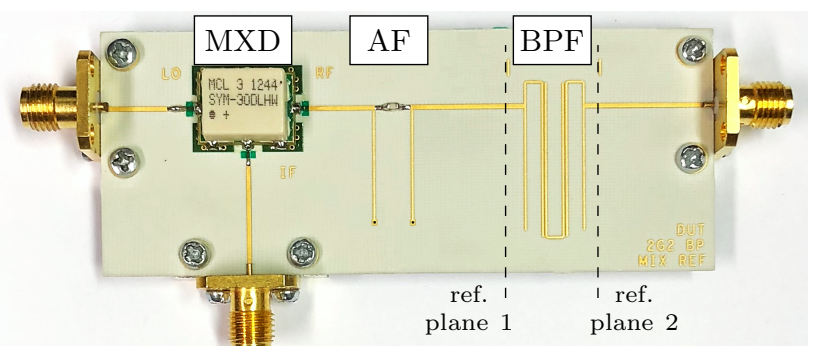

Fig. 5. Developed test board including (from left to right) a mixer, an auxiliary high-pass filter and measured BPF. 


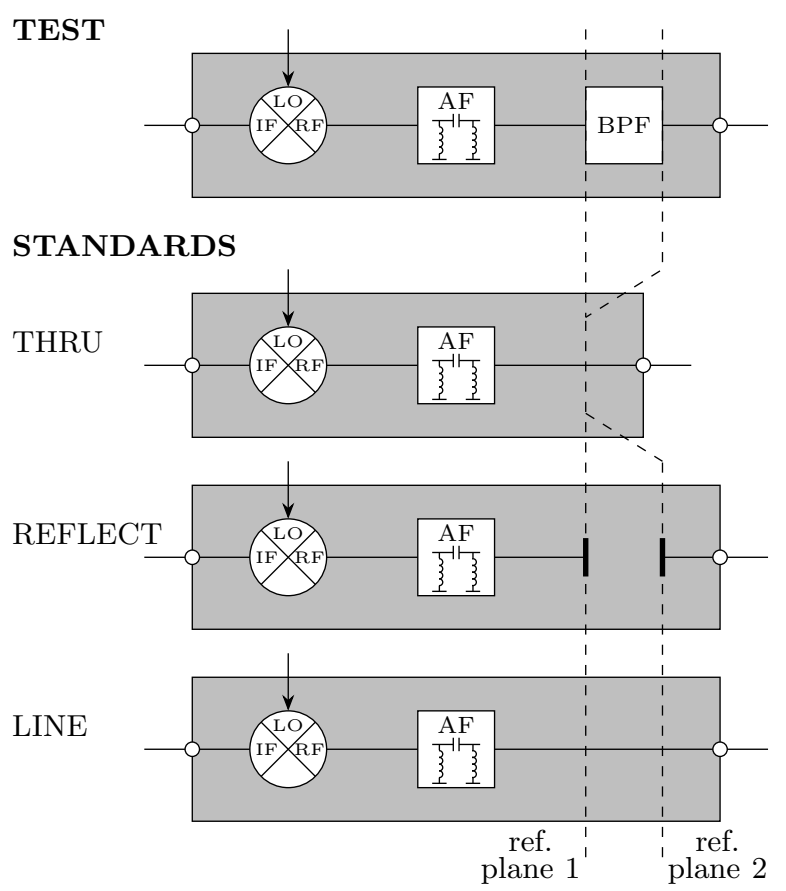

Fig. 6. Developed test and calibration boards.

In this case, this concerns, above all, the variances of the parameters of the mixers. For the given test, double balanced surface-mount SMD SYM-30DLHW (Mini-Circuits) modules were used. The obtained results show that their variances are very low and do not significantly influence the measurement. A more detailed uncertainty analysis is presented in Sec. 5.

\section{Practical Results}

The practical measurements were performed using a ZVA67 (0.01 - 67 GHz, Rohde-Schwarz) VNA. This unit includes two independently set LOs, so both LO1 and LO2 were internal. The VNA was pre-calibrated using the automatic short-open-load-thru (SOLT) module.

Figure 7 shows the measurement of the input reflection coefficient $\left(S_{11}\right.$ in $\left.\mathrm{dB}\right)$ of the embedded BPF in comparison with the measurement of the reference standalone filter. The measurement is affected by noticeable measurement error. The Peak at $2.1 \mathrm{GHz}$ is caused by the second harmonic of LO2 frequency generated in the external mixers and leaking into the VNA's reference and measurement channels.

Figures 8 and 9 depicts the results of similar $S_{12}$ ans $S_{21}$ measurements, respectively. As in previous case the peak at $2.1 \mathrm{GHz}$ is caused by the interfering second harmonic. The measurement accuracy for both $S_{12}$ and $S_{21}$ is satisfactory.

Figure 10 shows the results of the $S_{22}$ measurements. Compared to Fig. 7, its accuracy is significantly better. This can be explained by higher attenuation in the measurement branch and the variances of mixer parameters. The peak at $2.1 \mathrm{GHz}$ is once again caused by the interfering second harmonic.

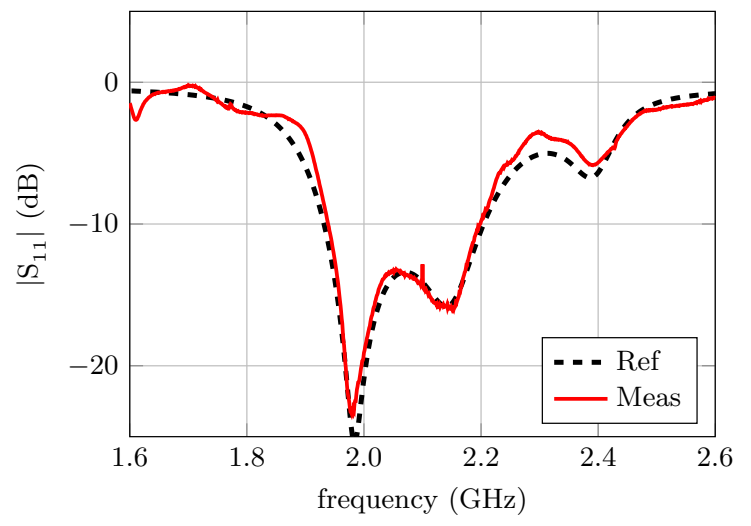

Fig. 7. Reflection coefficient $S_{11}$ of the measured BPF.

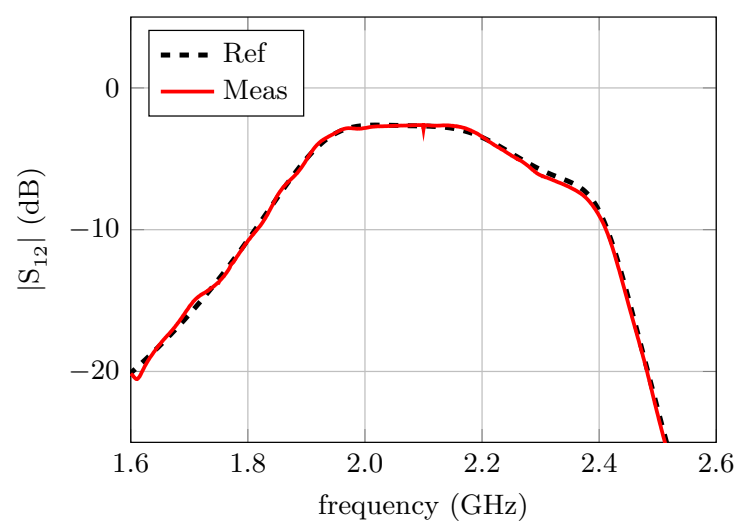

Fig. 8. Transmission coefficient $S_{12}$ of the measured BPF.

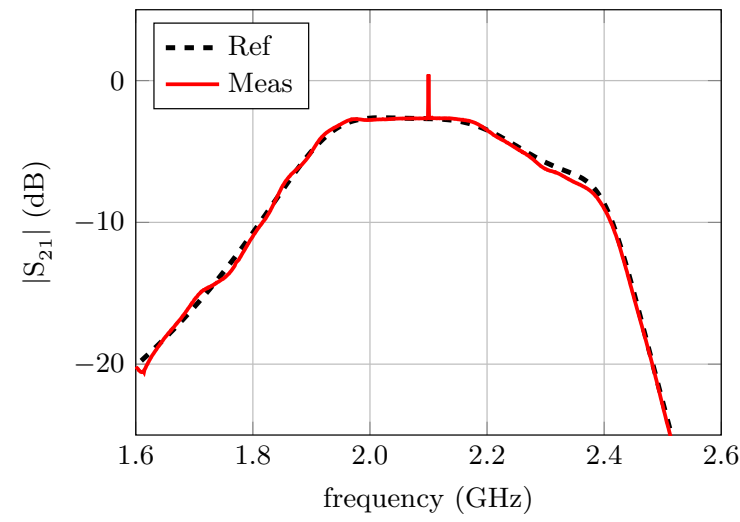

Fig. 9. Transmission coefficient $S_{21}$ of the measured BPF.

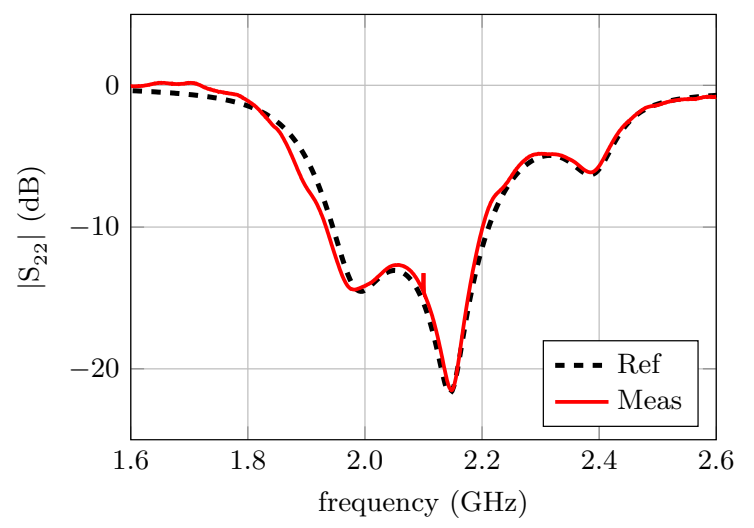

Fig. 10. Reflection coefficient $S_{22}$ of the measured BPF. 


\section{Uncertainty Analysis}

To determine confidence intervals and the accuracy of the aformentioned measurement method, an uncertainty analysis was performed. It takes into account variations of the S-parameters of the ERR-A error two-port consisting of the mixer, auxiliary filter and sections of the interconnecting microstrip lines as shown in Fig. 11. Since the ERR-B error two-port includes only a short section of the $50 \Omega$ line, its influence is neglected. Beforehand, to eliminate the influence of the rest of the measurement setup (measurement cables, adapters, external mixers and filters), the VNA was virtually calibrated in the PORT1-PORT2 planes, see Fig. 2.

As mentioned in Sec. 2, for the concerned uncertainty analysis, the complexity of equations used within the TRL calibration-correction algorithm complicates the use of the standard law of uncertainty propagation tool defined in Guide to the Expression of Uncertainty in Measurement (GUM) [12]. This method offers the most general approach, but, in this case, it alludes to an excess of variables with uncertain values as well as to analytical derivatives which are too complex, see [13]. To compute final uncertainty as defined in [14], the Monte-Carlo method was used.

The Monte-Carlo method is based on an analytical description of the circuit being studied and on knowledge of the variations and types of probability distributions of all individual components with uncertain values. If both items are available, it is possible to perform a high number of virtual measurements and to evaluate the resulting statistical data. An insufficient number of statistical properties of the used mixers were familiar, so the measured $S_{i j \mathrm{~A}} \mathrm{~S}$-parameters of the ERR-A error two-port were used and variations were applied to all of them. Owing to mutual relations among all $\mathrm{S}$-parameters (a change of the inner parameter of any twoport can result in changes to all of its S-parameters) this process leads to "worst-case" results, and real variances of DUT S-parameters are likely to be lower. The mean values of the variables in question (amplitudes, phases) correspond to the measured $S_{i j \mathrm{~A}}$ values and their standard deviation have been estimated to be $\sigma_{\mathrm{A}}=0.01$ using a Gaussian probability distribution. This $\sigma_{\mathrm{A}}$ value was obtained from the mixer's datasheet as a standard deviation of its conversion loss. The magnitudes of the ERR-A S-parameters in $\mathrm{dB}$ obtained from the THRU measurement can be seen in Fig. 12.

The error two-port described above contains four complex parameters which leads to eight real variables to be varied in the Monte-Carlo method. As $S_{i j \mathrm{D}}$ DUT $\mathrm{S}$-parameters, the values obtained by measuring the reference filter have been considered. Results of this analysis can be seen in Fig. 13, where magnitudes of the corrected DUT S-parameters are displayed.

The Fig. 12 depicts the magnitudes of the measured DUT S-parameters in bold lines, and confidence intervals of the given parameters are shown in the shaded regions. Confidence intervals are measured in units of $2 \sigma_{i j}$ where $\sigma_{i j}$ represents the standard deviation of the given S-parameter as calculated by the Monte-Carlo method.

Figure 13 indicates that $\left|S_{11 \mathrm{D}}\right|$ shows the highest sensitivity to ERR-A variations. This result is in good agreement with the measurement presented in Fig. 7 where $\left|S_{11 \mathrm{D}}\right|$ is obviously measured in the least accurate way. Other S-parameters are influenced substantially less and measurement accuracy seems to be fully satisfactory, especially in the most interesting filter band-pass frequency band. In spite of higher $S_{11 \mathrm{D}}$ variances, for our given purposes (a postmanufacturing check that the filter is tuned to the proper frequency band and that it shows acceptable RL return loss and IL insertion loss values), even this measurement can be considered as acceptable. Due to visible frequency ripples on the confidence interval dependences, it may be supposed that the DUT S-parameter variations depend on frequency, as shown in Fig. 13. In a similar way, the uncertainties of similar measurements can be evaluated. To investigate the reasons for this behavior, another uncertainty analysis was performed. As noted earlier, the TRL calibration-correction process does not allow the uncertainty analysis to be performed analytically. Assuming the ERR-B would behave as an ideal thru, an attempt was made to perform such an analysis on correction formulas applicable to the evaluation of $S_{i j \mathrm{D}}$ DUT S-parameters from the measured $S_{i j \mathrm{M}}$ S-parameters.

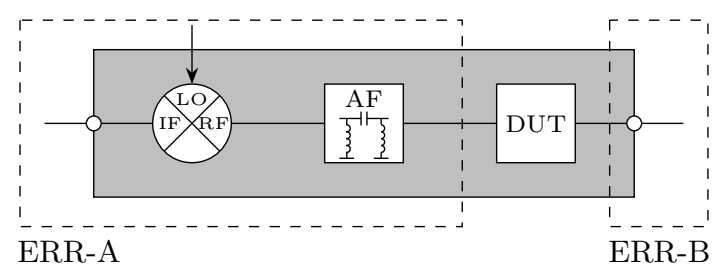

Fig. 11. Definition of Error boxes.

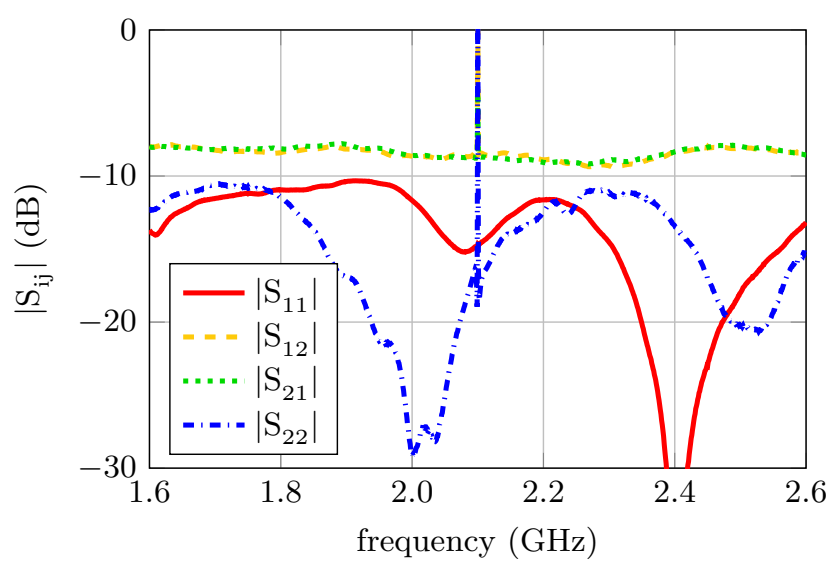

Fig. 12. Measured modules of S-parameters of the ERR-A error two-port. 


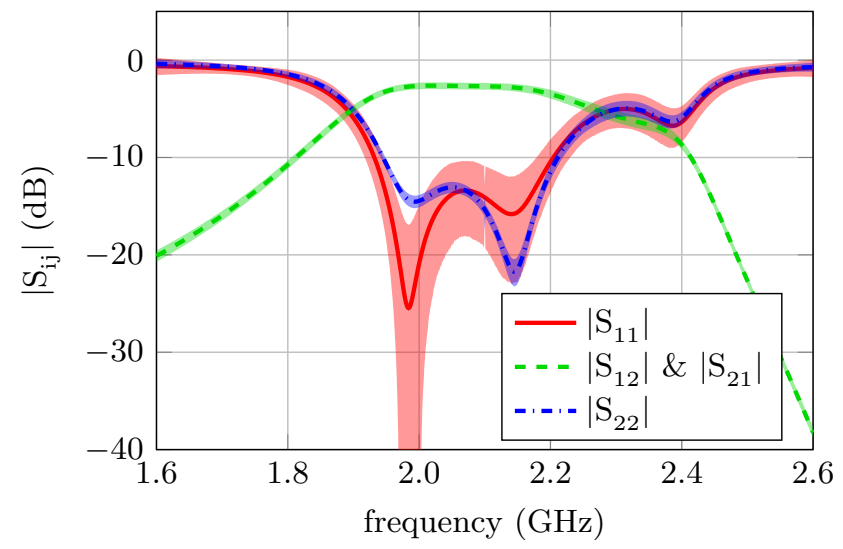

Fig. 13. Confidence intervals of the DUT measurement.

Considering a structure according to Fig. 11, the measured S-parameters can be expressed as

$$
\begin{gathered}
S_{11 \mathrm{M}}=S_{11 \mathrm{~A}}+S_{11 \mathrm{D}} \frac{S_{12 \mathrm{~A}} S_{21 \mathrm{~A}}}{1-S_{11 \mathrm{D}} S_{22 \mathrm{~A}}}, \\
S_{12 \mathrm{M}}=\frac{S_{12 \mathrm{D}} S_{12 \mathrm{~A}}}{1-S_{11 \mathrm{D}} S_{22 \mathrm{~A}}}, \\
S_{21 \mathrm{M}}=\frac{S_{21 \mathrm{D}} S_{21 \mathrm{~A}}}{1-S_{11 \mathrm{D}} S_{22 \mathrm{~A}}}, \\
S_{22 \mathrm{M}}=S_{22 \mathrm{D}}+S_{22 \mathrm{~A}} \frac{S_{12 \mathrm{D}} S_{21 \mathrm{D}}}{1-S_{11 \mathrm{D}} S_{22 \mathrm{~A}}} .
\end{gathered}
$$

From these equations, the correction formulas for the evaluation of the $S_{i j \mathrm{D}}$ DUT S-parameters can be expressed as

$$
\begin{aligned}
& S_{11 \mathrm{D}}=\frac{S_{11 \mathrm{~A}}-S_{11 \mathrm{M}}}{S_{22 \mathrm{~A}} S_{11 \mathrm{~A}}-S_{12 \mathrm{~A}} S_{21 \mathrm{~A}}-S_{22 \mathrm{~A}} S_{11 \mathrm{M}}}, \\
& S_{12 \mathrm{D}}=\frac{S_{12 \mathrm{M}} S_{21 \mathrm{~A}}}{S_{22 \mathrm{~A}} S_{11 \mathrm{~A}}-S_{12 \mathrm{~A}} S_{21 \mathrm{~A}}-S_{22 \mathrm{~A}} S_{11 \mathrm{M}}}, \\
& S_{21 \mathrm{D}}=\frac{S_{21 \mathrm{M}} S_{12 \mathrm{~A}}}{S_{22 \mathrm{~A}} S_{11 \mathrm{~A}}-S_{12 \mathrm{~A}} S_{21 \mathrm{~A}}-S_{22 \mathrm{~A}} S_{11 \mathrm{M}}},
\end{aligned}
$$

$$
\begin{array}{r}
S_{22 \mathrm{D}}=\frac{S_{22 \mathrm{M}}\left(S_{12 \mathrm{~A}} S_{21 \mathrm{~A}}+S_{22 \mathrm{~A}}\left(S_{11 \mathrm{M}}-S_{11 \mathrm{~A}}\right)\right)}{S_{22 \mathrm{~A}} S_{11 \mathrm{~A}}-S_{12 \mathrm{~A}} S_{21 \mathrm{~A}}-S_{22 \mathrm{~A}} S_{11 \mathrm{M}}}- \\
\frac{S_{21 \mathrm{~A}} S_{12 \mathrm{M}} S_{22 \mathrm{~A}}}{S_{22 \mathrm{~A}} S_{11 \mathrm{~A}}-S_{12 \mathrm{~A}} S_{21 \mathrm{~A}}-S_{22 \mathrm{~A}} S_{11 \mathrm{M}}}
\end{array}
$$

The desired measurement variances as a function of the ERR-A S-parameters can be expressed by means of Jacobian matrices $\mathbf{J}_{1}$ to $\mathbf{J}_{4}$ which include partial derivatives of the (5) to (8) functions with respect to individual $S_{i j \mathrm{~A}}$ parameters. Using (1) to (4), $\mathbf{J}_{1}$ to $\mathbf{J}_{4}$ can be written as

$$
\begin{gathered}
\mathbf{J}_{1}=\frac{\partial S_{11 \mathrm{D}}}{\partial S_{i j \mathrm{~A}}}=\left(\begin{array}{cc}
-\frac{\left(1-S_{11 \mathrm{D}} S_{22 \mathrm{~A}}\right)^{2}}{S_{12 \mathrm{~A}} S_{21 \mathrm{~A}}} & \frac{S_{11 \mathrm{D}}\left(1-S_{11 \mathrm{D}} S_{22 \mathrm{~A}}\right)}{S_{12 \mathrm{~A}}} \\
\frac{S_{11 \mathrm{D}}\left(1-S_{11 \mathrm{D}} S_{22 \mathrm{~A}}\right)}{S_{21 \mathrm{~A}}} & -S_{11 \mathrm{D}}^{2}
\end{array}\right), \\
\mathbf{J}_{2}=\frac{\partial S_{12 \mathrm{D}}}{\partial S_{i j \mathrm{~A}}}=\left(\begin{array}{cc}
-\frac{S_{22 \mathrm{~A}}\left(1-S_{11 \mathrm{D}} S_{22 \mathrm{~A}}\right)}{S_{12 \mathrm{~A}} S_{21 \mathrm{~A}}} & \frac{1-S_{11 \mathrm{D}} S_{22 \mathrm{~A}}}{S_{12 \mathrm{~A}}} \\
\frac{S_{11 \mathrm{D}} S_{22 \mathrm{~A}}}{S_{21 \mathrm{~A}}} & -S_{11 \mathrm{D}}
\end{array}\right),
\end{gathered}
$$

$$
\mathbf{J}_{3}=\frac{\partial S_{21 \mathrm{D}}}{\partial S_{i j \mathrm{~A}}}=\left(\begin{array}{cc}
-\frac{S_{22 \mathrm{~A}}\left(1-S_{11 \mathrm{D}} S_{22 \mathrm{~A}}\right)}{S_{12 \mathrm{~A}} S_{21 \mathrm{~A}}} & \frac{S_{11 \mathrm{D}} S_{22 \mathrm{~A}}}{S_{12 \mathrm{~A}}} \\
\frac{1+S_{11 \mathrm{D}} S_{22 \mathrm{~A}}}{S_{21 \mathrm{~A}}} & -S_{11 \mathrm{D}}
\end{array}\right),
$$

$$
\mathbf{J}_{4}=\frac{\partial S_{22 \mathrm{D}}}{\partial S_{i j \mathrm{~A}}}=S_{12 \mathrm{D}} S_{21 \mathrm{D}}\left(\begin{array}{cc}
-\frac{S_{22 \mathrm{~A}}^{2}}{S_{12 \mathrm{~A}} S_{21 \mathrm{~A}}} & \frac{S_{22 \mathrm{~A}}}{S_{12 \mathrm{~A}}} \\
\frac{S_{22 \mathrm{~A}}}{S_{21 \mathrm{~A}}} & -1
\end{array}\right)
$$

To ensure the minimal influence of variations of ERR-A on the DUT measurement, the modules of terms in the Jacobian matrices (9) to (12) should also be as low as possible. In (9) to (12), the majority of numerators include the following common term

$$
f=1-S_{11 \mathrm{D}} S_{22 \mathrm{~A}} \text {. }
$$

Thanks to the multiplication of $S_{11 \mathrm{D}}$ and $S_{22 \mathrm{~A}}$, this term depends on the sum of their phases. Therefore, it is possible to distinguish two important states providing the highest and lowest values of (13). For absolutely stable devices, the magnitudes of the $S_{11 \mathrm{D}}$ and $S_{22 \mathrm{~A}}$ are always $\leq 1$ and the absolute value of their product is also $\leq 1$. This results in an absolute value of (13) being in interval $(0,2)$. The term approaches number 0 when

$$
\exp \left[\mathrm{j}\left(\angle S_{11 \mathrm{D}}+\angle S_{22 \mathrm{~A}}\right)\right]=1,
$$

while it approaches number 2 when

$$
\exp \left[\mathrm{j}\left(\angle S_{11 \mathrm{D}}+\angle S_{22 \mathrm{~A}}\right)\right]=-1
$$

This corresponds to $\angle S_{22 \mathrm{~A}}+\angle S_{11 \mathrm{D}}=2 n \pi \quad$ or $\angle S_{22 \mathrm{~A}}+\angle S_{11 \mathrm{D}}=(2 n+1) \pi$ respectively, where $\mathrm{n}$ is an integer. 


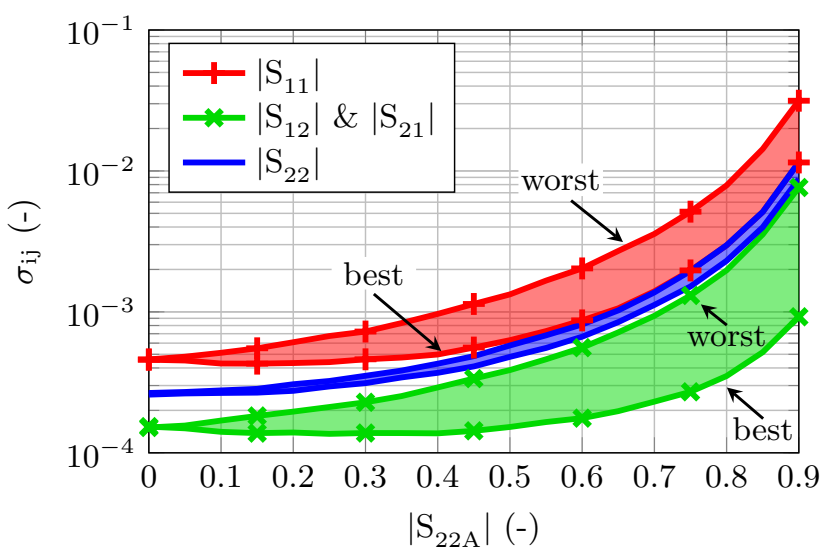

Fig. 14. Variances of $\left|S_{i j \mathrm{D}}\right|$ as function of $\left|S_{22 \mathrm{~A}}\right|$ and best and worst phasing conditions.

To evaluate the influences of the $S_{22 \mathrm{~A}}$ amplitude and phase on the DUT measurement using TRL techniques, another Monte-Carlo uncertainty analysis was performed. In each run, only a single frequency point was examined. As with the ERR-A error two-port, a synthetic symmetric and lossless two-port was used while its $\left|S_{22 \mathrm{~A}}\right|$ amplitude was changed as a parameter. For each set $\left|S_{22 \mathrm{~A}}\right|$ value, two ERR-A versions were synthesized with phasing corresponding to the best and worst cases described by (14) and (15). Consequently, as in the previous case, the ERR-A S-parameters were varied with a variance equal to 0.01 and Gaussian distribution. From numerous synthetic measurements performed by the Monte-Carlo procedure, $\sigma_{i j}$ variances corresponding to the $\left|S_{11 \mathrm{D}}\right|,\left|S_{22 \mathrm{D}}\right|$ and $\left|S_{21 \mathrm{D}}\right|$ parameters were evaluated.

The resulting $\sigma_{i j}$ values as a function of $\left|S_{22 \mathrm{~A}}\right|$, along with best or worst case phasing, can be seen in Fig. 14. For each DUT S-parameter, the dependence with higher $\sigma_{i j}$ values corresponds to the worst case while the dependence with lower $\sigma_{i j}$ values depicts the best case. The resulting $\sigma_{i j}$ uncertainty, therefore, will lie between these two plots. Since the (14) and (15) phasing conditions are frequency dependent, the $\sigma_{i j}$ variances are also frequency dependent, as can be seen in Fig. 13.

Figure 14 confirms that $\left|S_{11 \mathrm{D}}\right|$ is measured with the highest uncertainty and that there can appear a significant frequency ripple of its confidence interval frequency dependence. As expected, $\left|S_{22 \mathrm{D}}\right|$ variations are lower and show extremely low susceptibility to frequency dependent rippling, with the expected uncertainty of $\left|S_{21 \mathrm{D}}\right|$ being the lowest. All these results are in good agreement with the calculated frequency dependent confidence intervals shown in Fig. 13 and the measurements shown in Figs. 7, 9 and 10.

\section{Conclusion}

The article presents a description and the practical results of a new method enabling the measurement of individual components or circuits embedded in more complex RF or microwave PCBs containing frequency conversion. As an ex- ample, the planar microstrip filter embedded in a common upconverting module was measured. Included plots show good agreement between results obtained by the proposed method and the direct measurement of the reference filter. The developed method can, therefore, be used for "non-destructive" measurements of similarly manufactured RF and microwave modules.

The uncertainty analysis shows that the results obtained by the method presented can be fully applicable for the intended purposes, that means, for the final checkout of a microstrip filter etched at the RF system PCB. According to the simulations performed in the case presented, the $\left|S_{11 \mathrm{D}}\right|$ is the parameter most influenced by the variances of the $S$ parameters of the adjunct circuits. The expected variances of the other DUT S-parameters are significantly lower. This is in extremely good agreement with all measurements. The developed uncertainty analysis techniques can be used to predict DUT variances of any similar measurement setup.

Future developments will focus on a closer elaboration of the T-method, based on a direct parallel connection of special probes and the elimination of their influences through a similar calibration-correction process.

\section{Acknowledgments}

This work was supported by the Grant Agency of the Czech Technical University in Prague, grant No. SGS16/226/OHK3/3T/133.

\section{References}

[1] RENBI, A., RISSEH, A., QVARNSTROM, R., et al. Impact of etch factor on characteristic impedance, crosstalk and board density. In International Symposium on Microelectronics (IMAPS). San Diego (USA), 2012, vol. 2012, no. 1, p. 312-317. DOI: 10.4071 isom-2012-TP24

[2] TARATEERASETH, V., SEE, K. Y., CANAVERO, F. G., et al. Systematic electromagnetic interference filter design based on information from in-circuit impedance measurements. IEEE Transactions on Electromagnetic Compatibility, 2010, vol. 52, no. 3, p. 588-598. DOI: 10.1109/TEMC.2010.2046419

[3] FORGACS, R. L. In-circuit impedance measurement using current sensing. IEEE Transactions on Instrumentation and Measurement, 1985, vol. IM-34, no. 1, p. 6-14. DOI: 10.1109/TIM.1985.4315246

[4] ZELDER, T., GECK, B. Contactless scattering parameter measurements. IEEE Microwave and Wireless Components Letters, 2011, vol. 21, no. 9, p. 504-506. DOI: 10.1109/LMWC.2011.2162619

[5] STENARSON, J., YHLAND, K., WINGQVIST, C. An in-circuit noncontacting measurement method for s-parameters and power in planar circuits. IEEE Transactions on Microwave Theory and Techniques, 2001, vol. 49, no. 12, p. 2567-2572. DOI: $10.1109 / 22.971651$

[6] OUREDNIK, P., ADLER, V., HUDEC, P. TRL-based measurement of active antennas and other more complex microwave structures. In Proceedings of the 89th Microwave Measurement Conference (ARFTG). Honololu (USA), 2017, p. 1-4. DOI: 10.1109/ARFTG.2017.8000834 
[7] DUNSMORE, J.P. Handbook of Microwave Component Measurements: With Advanced VNA Techniques. Wiley, 2012. ISBN: 978-1-119-97955-5

[8] ANDERSON, K., HAWKINS, R., LUI, J., et al. Vector Network Analyzer with Independently Tuned Receivers Characterizes Frequency Translation Devices. U.S. Patent No. 7,248,033. 24 Jul. 2007.

[9] ROHDE \& SCHWARZ. R\&S ZVA / R\&S ZVB / R\&S ZVT Vector Network Analyzers Operating Manual. 1185 pages. [Online] Cited 2018-06-10. Available at: https://cdn.rohdeschwarz.com/pws/dl_downloads/dl_common_library/dl_manuals/ gb_1/z/zva_2/ZVA_ZVB_ZVT_OperatingManual_en_30.pdf

[10] ENGEN, G. F., HOER, C. A. Thru-Reflect-Line: An improved technique for calibrating the dual six-port automatic network analyzer. IEEE Transactions on Microwave Theory and Techniques, 1979, vol. 27, no. 12, p. 987-993. DOI: 10.1109/TMTT.1979.1129778

[11] TEPPATI, V., FERRERO, A., SAYED, M. Modern RF and Microwave Measurement Techniques. Cambridge University Press, 2013. ISBN: 9781107036413

[12] JOINT COMMITTEE GUIDES METROLOGY. Evaluation of Measurement Data - Guide to the Expression of Uncertainty in Measurement. 134 pages. [Online] Cited 2018-06-10. Available at: https://www.bipm.org/utils/common/documents/jcgm/JCGM 100_2008_E.pdf

[13] LINZCZUK, P., ZDUNEK, P., BARMUTA, P., et al. GPU implementation of multiline TRL calibration for efficient Monte-Carlo uncertainty analysis. In Proceedings of the 21 st International Conference on Microwave, Radar and Wireless Communications (MIKON). Krakow (Poland), 2016, p. 1-4. DOI: 10.1109/MIKON.2016.7492106

[14] JOINT COMMITTEE GUIDES METROLOGY. Evaluation of Measurement Data - supplement 1 to the "Guide to the Expression of Uncertainty in Measurement"- Propagation of Distributions Using a Monte Carlo Method. 90 pages. [Online] Cited 2018-06-10. Available at: https://www.bipm.org/utils/common/documents/jcgm/ JCGM_101_2008_E.pdf

\section{About the Authors ...}

Petr OUREDNIK was born in Prague, Czech Republic, in 1991. He received the M.S. degree in Wireless Communication in 2016 from the Czech Technical University in Prague, Czech Republic, where he is currently working toward the $\mathrm{Ph} . \mathrm{D}$. degree in Radioelectronics. His current research interests include microwave measurement and radar sensor for fast traveling targets. $\mathrm{He}$ is also interested in the design and measurement of passive and active microwave circuits, electromagnetic simulators, and uncertainty analyses.

Premysl HUDEC received the M.S. and Ph.D. degrees in Electrical Engineering from the Czech Technical University in Prague, Czech Republic, in 1982 and 1995, respectively. Since 2010, he has been working as an Associate Professor in the Department of the Electromagnetic Field, Czech Technical University in Prague. His teaching duties include CAD of microwave circuits and microwave measurements. In recent years, his research activities have been focused on the employment of microwave technology in security applications. Primarily, these works include the design of different types of microwave radar sensors for active defense systems and the development of RFID systems for long-range identification. He is the author of numerous conference papers, journal articles, and patents 